\title{
Una propuesta didáctica de las ecuaciones diferenciales en el contexto de la ingeniería de control
}

\section{A didactic proposal of differential equations in the context of control engineering}

\author{
SAUZA-TOLEDO, Martín†**, RESENDÍZ-RÍOS, Juan, VEGA-GARCÍA, Raquel y BUITRÓN-
} RAMÍREZ, Hugo Armando

Universidad Tecnológica de Tula-Tepeji-Cuerpo Académico Matemáticas aplicadas a proyectos tecnológicos y su didáctica -Cuerpo Académico Emprendedurismo. venida Universidad Tecnológica, El Carmen, 42830 Hgo.

ID 1 ${ }^{\text {er }}$ Autor: Martín, Sauza-Toledo / ORC ID: 0000-0002-7860-4221, CVU CONACYT ID: 686916

ID 1 $1^{\text {er }}$ Coautor: Juan, Resendíz-Ríos / ORC ID: 0000-0002-3122-2621, CVU CONACYT ID: 307843

ID $2^{\text {do }}$ Coautor: Raquel, Vega-García / ORC ID: 0000-0001-8074-2685

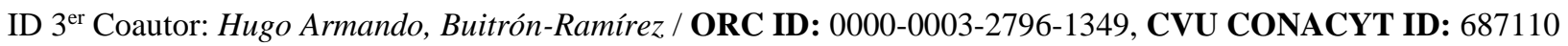

DOI: $10.35429 /$ JPD.2019.9.3.14.18

Recibido 01 de Agosto, 2019; Aceptado 29 de Septiembre, 2019

\section{Resumen}

El presente trabajo, pretende rendir cuenta de las investigaciones realizadas en el contexto de las matemáticas en la ingeniería de control, específicamente en el área de la ingeniería eléctrica y electrónica. Por lo anterior, en este artículo se muestra la deficiente aplicación de las matemáticas, en el entorno industrial, específicamente en las ecuaciones diferenciales, de igual manera se justifica la pertinencia del tema de estudio dentro del área de la Matemática Educativa y se describen los antecedentes necesarios, para hacer una propuesta didáctica de las ecuaciones diferenciales en las líneas de transmisión. La investigación esta soportada en el constructivismo, aprendizaje significativo y la matemática en el contexto de las ciencias. El cual es resultado de indagar que tan profundas son las aplicaciones en el entorno industrial, de acuerdo a los estudios realizados en distintas empresas de la región Tula-Tepeji, con la ayuda de un instrumento de investigación exploratorio aplicado directamente a ingenieros en ejercicio. Por lo anterior se considera pertinente resaltar que este trabajo es resultado de la línea de investigación que cultiva el cuerpo académico.

Matemática Educativa, Matemática en Contexto, Discurso Matemático Escolar, Propuesta Didáctica

\begin{abstract}
The present work tries to give account of the investigations realized in the context of the mathematics in the engineering of control, specifically in the area of the electrical and electronic engineering. Therefore, this article shows the poor application of mathematics, in the industrial environment, specifically in differential equations, the relevance of the subject of study within the area of Educational Mathematics is justified and the background is described. Necessary, to make a didactic proposal of the differential equations in the transmission lines. Research is supported by constructivism, meaningful learning and mathematics in the context of science. Which is the result of investigating how deep the applications are in the industrial environment, according to studies conducted in different companies in the TulaTepeji region, with the help of an exploratory research instrument applied directly to engineers in practice. Therefore, it is considered pertinent to highlight that this work is the result of the line of research cultivated by the academic body.
\end{abstract}

Educational Math, Math in Context, School Math Speech, Didactic Proposal

Citación: SAUZA-TOLEDO, Martín, RESENDÍZ-RÍOS, Juan, VEGA-GARCÍA, Raquel y BUITRÓN-RAMÍREZ, Hugo Armando. Una propuesta didáctica de las ecuaciones diferenciales en el contexto de la ingeniería de control. Revista de Didáctica Práctica. 2019. 3-9: 14-18.

\footnotetext{
*Correspondencia al Autor (Correo Electrónico: msauza73@ @otmail.com)

$\dagger$ Investigador contribuyendo como primer autor.
} 


\section{Introducción}

El presente trabajo de investigación es resultado de indagar que tan profundas son las aplicaciones en el entorno industrial, de acuerdo a un estudio realizado en distintas empresas de la región Tula-Tepeji y con la ayuda de algunos cuestionarios de exploración aplicados directamente a ingenieros en ejercicio, se obtuvieron los siguientes datos y gráficas, en donde se muestran que tanto aplican matemáticas y los temas en particular que los ocupa como profesionistas e ingenieros en ejercicio.

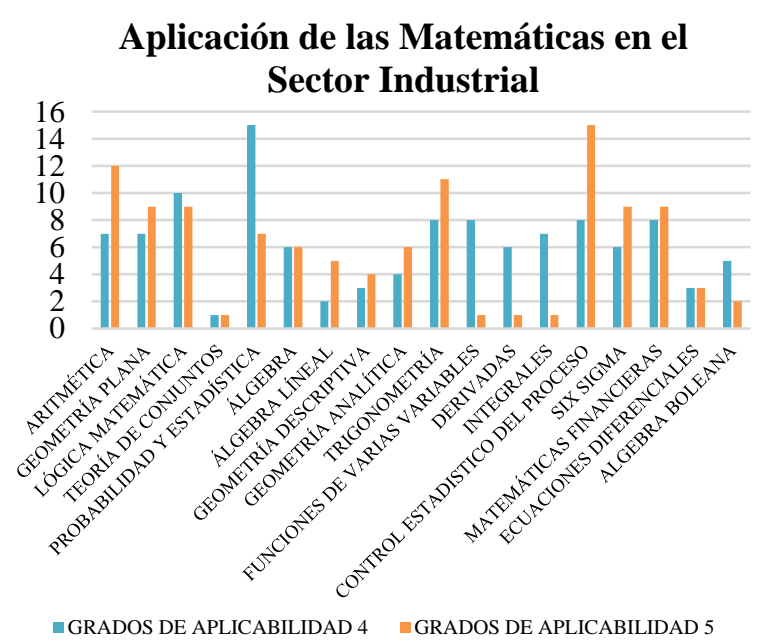

Gráfico 1 Aplicación de Matemáticas

Como podemos observar en la gráfica 1 , en el sector industrial, los ingenieros hacen mayor uso de probabilidad y estadística, así como control estadístico del proceso; en segunda instancia está el uso de la aritmética, trigonometría, geometría plana, lógica matemática, seis sigma y matemáticas financieras.

De los resultados anteriormente expuestos, podemos observar, que existe una necesidad de contextualizar las ecuaciones diferenciales, a través de problemas cuyo origen se fundamente de problemas reales, más en el área de diseño.

Es en esta línea donde vemos muy pertinente cultivar la línea de investigación de las ecuaciones diferenciales en el contexto de la ingeniería, siendo más específicos en la ingeniería de control.
Por otro lado, no podemos soslayar que una parte importante, de la aplicación de las matemáticas, es el rigor en los procedimientos y el tratamiento algebraico, que requieren las ecuaciones diferenciales, sin olvidar que el cálculo diferencial e integral juega un papel importante en los procesos cognitivos para la construcción de este conocimiento, es importante, resaltar entonces que estos procesos cognitivos son los que hace al ingeniero en ejercicio analítico y reflexivo ante los problemas a contextualizar.

\section{Justificación}

De acuerdo con Sauza, M. (2007) los fenómenos didácticos que se identifican en el aula, referentes a la relación profesor-alumno, compete a la Matemática Educativa enfatizar en el tratamiento y soporte metodológico, en donde se fundamente el estudio y con ello poder caracterizar el problema, desde el punto de vista educativo se detectan algunas áreas de oportunidad en el aula, derivado de que los alumnos no contextualizan lo aprendido en sus cursos curriculares y por ende no tienen la necesidad de aplicar lo aprendido como una herramienta, para la solución de problemas. Con lo anterior, se determina la transposición del saber a enseñar al saber de aplicación; la cual se ha denominado, transposición generalizada (Camarena, P. 2000).

Es importante enfatizar que las herramientas matemáticas, juegan un papel importante en el estudio y modelación matemática de los sistemas a controlar, algunas de estas aplicaciones se pueden ver en La Transformada Rápida de Fourier como elemento para mediar señales en tiempo real (Sauza, M. 2014), muestra la transformada rápida de Fourier como una herramienta para medir y controlar señales en tiempo real, cabe destacar que sin el contexto de esta herramienta en la ingeniería de control, no fuera posible modelar y medir las señales en estudio. Así mismo, Camarena (1990, p. 16) detalla que la electrónica tiene injerencia en todos los campos científicos, económicos, humanísticos: a través de instrumentos de medición, computadoras, máquinas para la industria, aparatos caseros, juegos o, a través de la diversificación satelital, las telecomunicaciones, la acústica, el control electrónico, la sismografía, la bioelectrónica, la robótica, el hardware, la telefonía. 
Si bien la matemática educativa, es una disciplina, creada para abordar los fenómenos didácticos que se dan en el proceso de enseñanza de las matemáticas, también es menester de esta disciplina influir en el discurso matemático escolar y en la curricula de las áreas del conocimiento donde es indispensable la aplicación de las matemáticas.

Toca entonces a los matemáticos educativos, afectar los programas de estudio y el discurso matemático escolar a través de propuestas didácticas, que tengan injerencia directamente en el salón de clase.

De acuerdo con Cantoral, R. (2001) Uno de los objetivos de la matemática educativa, es reconocer estrategias que favorecen la construcción de conocimiento matemático.

Derivado de lo anterior, es menester de los profesores que imparten matemáticas en escuelas de ingeniería enfatizar en ejercicios de aplicación. Mostrar las aplicaciones de la teoría en problemas reales, es decir contextualizar lo aprendido a través de ejercicios con aplicaciones.

\section{Planteamiento del Problema}

La aplicación de las Matemáticas, como una herramienta para el ingeniero en ejercicio en la resolución de problemas.

\section{Hipótesis}

Son las matemáticas una herramienta del ingeniero en ejercicio en las distintas ramas de la ingeniería.

\section{Objetivo General}

Indagar hasta donde son aplicables las matemáticas como herramienta para la solución de problemas en el entorno industrial.

\section{Objetivos específicos}

Estudio preliminar de la aplicabilidad de las matemáticas en las industrias de la región TulaTepeji, a través de una encuesta a los ingenieros en ejercicio.

Análisis e interpretación de los resultados obtenidos de la encuesta.

\section{Propuesta Metodológica}

Derivado de lo anterior, se muestra un ejemplo de aplicación en el contexto de ingeniería de control bajo la siguiente propuesta, la cual se detalla a continuación:

a) Caracterización: Identificar las variables que intervienen en un proceso de control determinado.

b) Contextualización: Buscar las ecuaciones matemáticas que ayuden a describir las variables identificadas, que permitirán contextualizar el problema de estudio.

c) Modelación: Obtener el modelo matemático que describe las variables que están interactuando, ya contextualizadas.

d) Resolver matemáticamente el problema, aplicando algún método conocido para la solución de ecuaciones diferenciales.

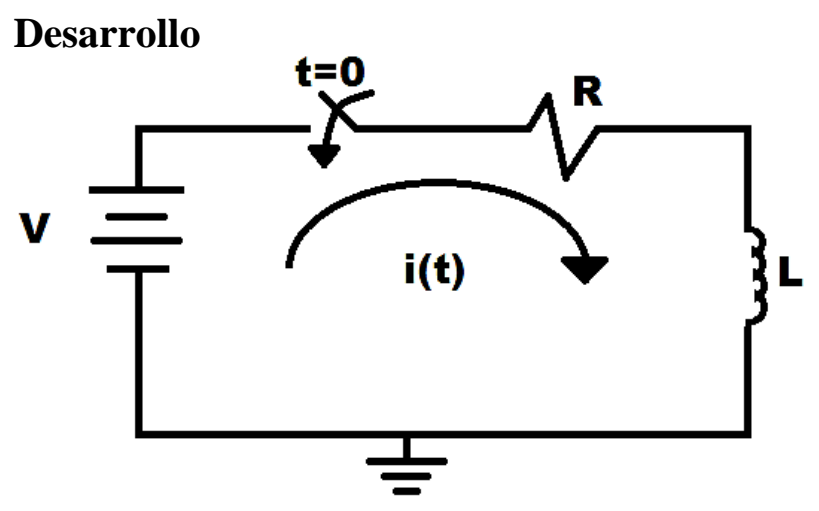

Figura 1 Circuito eléctrico

La figura 1, muestra un circuito electrico, el cual es también un sistema de primer orden, el sistema de primer orden esta formado en este caso en particular por un inductor $L$, el cual es un elemento que almacena energia, y la resistencia $R$, la cual se opone al flujo de electrones (corriente electrica).

El voltaje en una resistencia, es igual a la resistencia, por la corriente que circula a traves de ella y se representa con la siguiente ecuación:

$V_{R}=R i(t)$

De la misma manera, el voltaje en un inductor es igual al valor del inductor, por el cambio de la corriente con respecto al tiempo, es decir: 
$V_{L}=\frac{L d i(t)}{d t}$

De lo anterior y de acuerdo a las leyes de voltaje de Kirchoff, decimos que el voltaje de entrada es igual a la suma del voltaje en la resistencia, más el voltaje en el inductor.

$V=V_{R}+V_{L}$

Ahora sustituimos el valor de los voltajes en la ecuación anterior y aplicamos el método de variables separables, para resolverla.

$V=R i(t)+L \frac{d i(t)}{d t}$

\section{$V, R, L=$ Constantes}

Multiplicamos la ecuación $V=R i(t)+$ $L \frac{d i(t)}{d t}$ por $d t$ para darle la forma de la ecuación general de primer orden y primer grado

$M(x, y) d x+N(x, y) d y=0$

$V d t=R i(t) d t+L d i(t)$

$R i(t) d t-v d t+L d i(t)=0$

Factorizamos $d t$

$[\operatorname{Ri}(t)-v] d t+\operatorname{Ldi}(t)=0]$

Obtenemos el factor integrante.

$\alpha(t, i(t))=\frac{1}{R i(t)-v}$

$\frac{1}{R i(t)-v}([R i(t)-v] d t+L d i(t)=0)$

$\frac{\operatorname{Ri}(t)-v}{\operatorname{Ri}(t)-v} d t+\frac{L d i(t)}{\operatorname{Ri}(t)-v}=0$

$d t+\frac{L}{R i(t)-v} d i(t)=0$

$\int d t+\frac{L}{R} \int \frac{[R d i(t)]}{R i(t)-v}=c$

$t+\frac{L}{R} \ln (R i(t)-v)=c$

$\frac{L}{R} \ln (R i(t)-v)=c-t$

$$
\begin{aligned}
& \ln (R i(t)-v)=(c-t) \frac{R}{L} \\
& e^{\ln (R i(t)-v)}=e^{(C-t) \frac{R}{L}} \\
& (R i(t)-v)=\left(e^{C \frac{R}{L}}\right)\left(e^{-\frac{R}{L} t}\right) \\
& i(t)=\frac{V}{R}+\left(e^{C^{C} \frac{R}{L}}\right)\left(e^{-\frac{R}{L} t}\right) \\
& i(t)=\frac{V}{R}+c e^{-\frac{R}{L}} \\
& i(t)=\frac{V}{R}-\frac{V}{R} e^{-\frac{R}{L} t} \\
& \boldsymbol{i}(\boldsymbol{t})=\frac{\boldsymbol{V}}{\boldsymbol{R}}\left(\mathbf{1}-\boldsymbol{e}^{-\frac{\boldsymbol{R}}{L} t}\right)
\end{aligned}
$$

\section{Resultados}

Con la propuesta metodológica anteriormente expuesta, pretendemos mostrar que la enseñanza de las matemáticas, debe de partir de un problema real, donde el estudiante de ingeniería principalmente, vea la necesidad de contextualizar y aplicar las matemáticas como una herramienta del ingeniero en ejercicio en las distintas áreas de su competencia.

\section{Agradecimientos}

Mi agradecimiento al Ing. Adiel Basurto Guerrero y al M. en I. Juan Reséndiz Ríos, con quienes comparto el gusto por las matemáticas.

Agradezco a la Universidad Tecnológica de Tula Tepeji, por ser mi segunda casa, a la Rectora Dra. Irasema E. Linares Medina, por su apoyo y facilidades brindadas para llevar a buen término este proyecto.

Investigadores de instituciones internacionalmente reconocidas, de manera especial a la Dra. Patricia Camarena Gallardo del Instituto Politécnico Nacional (IPN), quien dedicó tiempo valioso para guiar el trabajo de investigación. 


\section{Conclusiones}

Los estudios preliminares que he desarrollado al realizar mi tesis para obtener el grado de Maestría en Ciencias con Orientación en la Enseñanza de las Matemáticas, me llevaron a concluir, que es necesaria la vinculación de las matemáticas con problemas reales, de ahí que surgió la propuesta ante el programa para el Desarrollo Profesional Docente para el tipo Superior (PRODEP), del proyecto denominado: Estudio de las aplicaciones matemáticas en el entorno industrial, en la región de Tula - Tepeji en el estado de Hidalgo, con el cual obtuve el financiamiento para la aplicación y publicación de un libro titulado Ecuaciones Diferenciales con ISBN: 978-607-96568-8-1.

\section{Referencias}

Ausubel, D. (1990). Psicología educativa, un punto de vista cognoscitivo. México: Trillas.

Camarena P, G. (2008). Actas del III Coloquio Internacional sobre Enseñanza de las Matemáticas. Teoría de la Matemática en el Contexto de las Ciencias. Peru.

Camarena P, G. (2013). A treinta años de la teoría educativa "Matemática en el contexto de las ciencias". Innovación Educativa.

Camarena, P. (1990). Especialidad en docencia de la ingeniería matemática en electrónica. México D.F.: ESIME-IPN.

Camarena, P. (2001). Las Funciones Generalizadas en Ingeniería construcción de una alternativa didáctica. México: ANUIES, Colección Biblioteca de la educación Superior, Serie Investigación.

Cantoral, R. (2001). Matemática Educativa un estudio de la formación social de la analiticidad. México D. F.: Grupo Editorial Iberoamerica.

Imaz, C. (1987). ¿Qué es la matemática Educativa?. México: Memorias de la Primera Reunión Centroamericana y del Caribe sobre Formación de Profesores e Investigación en Matemática Educativa.
Piaget, J. (1991). Introducción a la epistemología genética: el pensamiento matemático. México D. F.: Paidós, Psicología Evolutiva.

Sauza, M. (2007). Una propuesta didáctica del análisis matemático en el contexto de la ingeniería de control. México: Tesis de maestría en ciancias con orientación en la enseñanza de las matemáticas (Universidad Autónoma del Estado de Hidalgo). 